\title{
Vilsmeier-Haack Transformations under Non Classical Conditions
}

\author{
Tasneem Mohammed ${ }^{1, *}$, Aejaz A. Khan', S. M. Shakeel Iqubal', Touseef Begum ${ }^{2}$ \\ ${ }^{1}$ Department of General Science, Ibn Sina National College for Medical Studies, Jeddah, Kingdom of Saudi Arabia \\ 2Department of Pharmaceutical Science, Ibn Sina National College for Medical Studies, Jeddah, Kingdom of Saudi Arabia
}

*Corresponding author: E-mail: drtasneem1431@gmail.com; Tel: (+966) 562367167

DOI: $10.5185 /$ amlett.2020.021476

Anisoles and Pyridines undergo acetylation and formylation under non-classical Vilsmeier-Haack conditions. The reactants are grinded vigorously in a mortar for about 25 to $30 \mathrm{~min}$ at room temperature with a pestle. The introduction of formyl and acetyl groups under solvent free conditions is a unique, fast and efficient method which has not been reported in literature so far.

\section{Introduction}

Formyl and acetyl group can be efficiently introduced in organic substrates by Vilsmeier-Haack reaction (VHR). The Vilsmeier-Haack reagent is one such mild reagent widely applied for ring closure, acylation, formylation and ring annulation [1-5]. This reaction can be easily carried out on aromatic and heteroaromatic compounds, leading to the formation of carboxaldehydes and ketones which are potent precursors for biologically active compounds [6,7]. Halomethyleniminium salt formed from N-N'-Dimethylformamide (DMF) or N-N'Dimethylacetamide (DMA) and Phosphoryl chloride $\mathrm{POCl}_{3}$ has captivated the interest of chemists.

In Green synthesis, economically simple and environmentally safe methods have been developed during the recent past $[\mathbf{8 , 9}$ ]. A perusal of recent reviews and publications in this field, signifies solvent free organic reactions [10-14]. Their mild reaction conditions, enhanced selectivity, and ease of handling are the main features which highlights that this process is not only simple; but also appeases both economical and environmental requirements by substituting the toxic solvents. Although numerous attempts were successfully carried out to use operationally mild materials as catalysts, not much consideration appears to have been diverted in the direction of solvent free VHR [15-21]. Over the past decade our group is actively engaged in employing an array of green components like micelles and non-conventional energy sources such as microwave and ultra sound to facilitate various organic conversions like nitration, cyclisation and VilsmeierHaack reactions [22]. The current research probes into one such activity, which investigates the possibility to achieve formylation and acetylation of certain anisoles and pyridines with Vilsmeier-Haack reagent under nonclassical conditions, by using a mortar and pestle to grind the reactants.

\section{Experimental}

\section{General}

Melting points are measured using Bio-Technics digital automatic melting point apparatus in an open capillary tube and are uncorrected. IR spectra were recorded on were recorded on G.C. FTIR using Varian Nicollet (USA) spectrophotometers. NMR spectra were recorded on Joel-FT NMR-100 Q spectrometer. V.G. Micro mass 7070 and Finnegan mal 1020 instruments was used to characterize and record the EI Mass Spectra. Glass plates coated with Loba Chemie's silica gel were used to perform thin layer chromatography (TLC) with ethyl acetate and hexane as eluent mixture.

\section{General procedure for Vilsmeier-Haack synthesis}

Vilsmeier-Haack reagent (0.015 moles) was added to organic substrate 1a and $4 \mathbf{a}(0.01 \mathrm{~mol})$ taken in a mortar and grinded with a pestle for about 25 to 30 minutes. Progress of the reaction was periodically monitored by thin layer chromatography. After completion, the reaction mixture was treated with 5\% sodium thio sulphate solution, followed by addition of dichloroethane (DCE). The organic layer was separated, dried over $\mathrm{Na}_{2} \mathrm{SO}_{4}$, evaporated under vacuum, and purified with column chromatography. This methodology has been successfully used in both formylation and acetylation reactions. Isolated products were distinguished by spectroscopic methods.

\section{Results and discussion}

To check the generality of the reaction an array of substituted Anisoles 1b-1f and Pyridines 4b-4d are used as substrates under classical Vilsmeier Haack reaction conditions as shown in Schemes $\mathbf{1}$ and $\mathbf{2}$ respectively. The products of formylation 2a-2f, 5a-5d and acetylation 3a-3f, 6a-6d were formed with ease and in a short time. The yields of major products are compiled in Tables 1 


\section{Advanced}

and 2 . The products were characterized by IR, ${ }^{1} \mathrm{H}-\mathrm{NMR}$, and Mass spectra as shown in Tables 3-6.

$$
\begin{aligned}
& \text { Scheme - } 1 \\
& \text { 1a: } \mathrm{X}=\mathrm{H} \\
& 1 \mathrm{~b}: \mathrm{X}=2-\mathrm{CH}_{3} \\
& 1 \mathrm{c}: \mathrm{X}=4-\mathrm{CH}_{3} \\
& \text { 1d: } \mathrm{X}=4-\mathrm{NO}_{2} \\
& \text { 1e: } \mathrm{X}=4-\mathrm{Cl}^{2}-30 \mathrm{~min}
\end{aligned}
$$

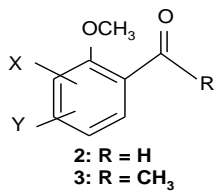

a: $\mathrm{X}=\mathrm{H}, \mathrm{Y}=4-\mathrm{OCH}_{3}$

b: $\mathrm{X}=3-\mathrm{CH}_{3}, \mathrm{Y}=4-\mathrm{OCH}_{3}$

c: $\mathrm{X}=2-\mathrm{CH}_{3}, \mathrm{Y}=5-\mathrm{OCH}_{3}$

$\mathrm{d}: \mathrm{X}=5-\mathrm{NO}_{2}, \mathrm{Y}=2-\mathrm{OCH}_{3}$

e: $\mathrm{X}=5-\mathrm{Cl}, \mathrm{Y}=2-\mathrm{OCH}_{3}$

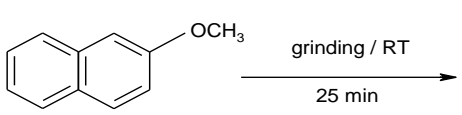<smiles>COc1ccc2ccccc2c1C(=O)O</smiles>

2f : $\mathrm{R}=\mathrm{H}$
$3 \mathrm{H}: \mathrm{R}=\mathrm{CH}_{3}$
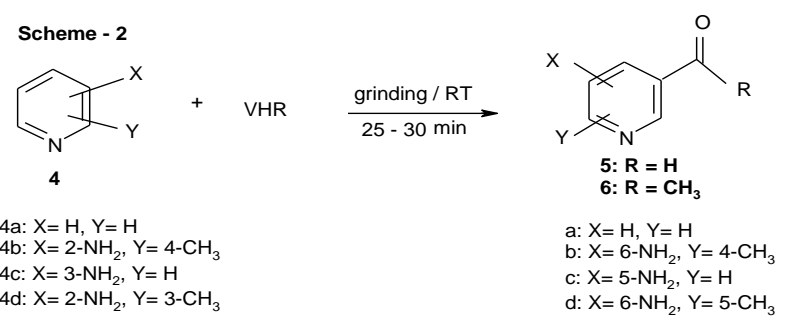

\begin{tabular}{|c|c|c|c|}
\hline Compound & $\mathbf{M}^{+}$ & IR $\left(\mathrm{Cm}^{-1}\right)$ & NMR \\
\hline $2 a$ & 136 & $1691(\mathrm{C}=\mathrm{O})$ & $\begin{array}{l}\delta 3.85\left(\mathrm{~s}, 3 \mathrm{H}, \mathrm{OCH}_{3}\right), \\
6.96(\mathrm{~d}, 2 \mathrm{H}, \mathrm{Ar}), \\
7.87(\mathrm{~d}, 2 \mathrm{H}, \mathrm{Ar}) \\
9.89(\mathrm{~s}, 1 \mathrm{H}, \mathrm{CHO})\end{array}$ \\
\hline $2 \mathbf{b}$ & 150 & $1689(\mathrm{C}=\mathrm{O})$ & $\begin{array}{l}2.16\left(\mathrm{~s}, 3 \mathrm{H}, \mathrm{CH}_{3}\right), \\
3.73(\mathrm{~s}, 3 \mathrm{H}, \mathrm{OCH})_{3}, \\
6.84(\mathrm{~d}, 1 \mathrm{H}, \mathrm{Ar}), \\
7.50(\mathrm{~d}, 1 \mathrm{H}, \mathrm{Ar}), \\
9.87(\mathrm{~s}, 1 \mathrm{H}, \mathrm{CHO})\end{array}$ \\
\hline $2 \mathrm{c}$ & 150 & $1691(\mathrm{C}=\mathrm{O})$ & $\begin{array}{l}\delta 2.35\left(\mathrm{~s}, 3 \mathrm{H}, \mathrm{CH}_{3}\right), \\
\left.3.73(\mathrm{~s}, 3 \mathrm{H}, \mathrm{OCH})_{3}\right) \\
6.84(\mathrm{~d}, 1 \mathrm{H}, \mathrm{Ar}) \\
7.23(\mathrm{~d}, 1 \mathrm{H}, \mathrm{Ar}) \\
7.50(\mathrm{~s}, 1 \mathrm{H}, \mathrm{Ar}) \\
10.24(\mathrm{~s}, 1 \mathrm{H}, \mathrm{CHO})\end{array}$ \\
\hline $2 d$ & 181 & $1690(\mathrm{C}=\mathrm{O})$ & $\begin{array}{l}83.73\left(\mathrm{~s}, 3 \mathrm{H}, \mathrm{OCH}_{3}\right), \\
7.22(\mathrm{~d}, 1 \mathrm{H}, \mathrm{Ar}) \\
8.48(\mathrm{~d}, 1 \mathrm{H}, \mathrm{Ar}) \\
8.63(\mathrm{~s}, 1 \mathrm{H}, \mathrm{Ar}) \\
10.24(\mathrm{~s}, 1 \mathrm{H}, \mathrm{CHO})\end{array}$ \\
\hline $2 \mathrm{e}$ & 170 & $1690(\mathrm{C}=\mathrm{O})$ & $\begin{array}{l}83.73\left(\mathrm{~s}, 3 \mathrm{H}, \mathrm{OCH}_{3}\right), \\
7.26(\mathrm{~d}, 1 \mathrm{H}, \mathrm{Ar}), \\
7.64(\mathrm{t}, 1 \mathrm{H}, \mathrm{Ar}) \\
10.24(\mathrm{~s}, 1 \mathrm{H}, \mathrm{CHO})\end{array}$ \\
\hline $2 f$ & 186 & $1673(\mathrm{C}=\mathrm{O})$ & $\begin{array}{l}83.73\left(\mathrm{~s}, 3 \mathrm{H}, \mathrm{OCH}_{3}\right), \\
7.16(\mathrm{~s}, 1 \mathrm{H}, \mathrm{Ar}), \\
7.48(\mathrm{t}, 1 \mathrm{H}, \mathrm{Ar}), \\
7.57(\mathrm{t}, 1 \mathrm{H}, \mathrm{Ar}), \\
7.60(\mathrm{~d}, 1 \mathrm{H}, \mathrm{Ar}) \\
7.64(\mathrm{~d}, 1 \mathrm{H}, \mathrm{Ar}), \\
8.15(\mathrm{~s}, 1 \mathrm{H}, \mathrm{Ar}), \\
10.24(\mathrm{~s}, 1 \mathrm{H}, \mathrm{CHO})\end{array}$ \\
\hline
\end{tabular}

4a: $\mathrm{X}=\mathrm{H}, \mathrm{Y}=\mathrm{H}$

$4 \mathrm{~b}: \mathrm{X}=2-\mathrm{NH}_{2}, \mathrm{Y}=4-\mathrm{CH}_{3}$

4c: $X=3-\mathrm{NH}_{2}, Y=\mathrm{H}$

$4 \mathrm{~d}: \mathrm{X}=2-\mathrm{NH}_{2}, \mathrm{Y}=3-\mathrm{CH}_{3}$

Table 3. Spectroscopic data of Formylation ${ }^{\mathrm{a}}$ Reactions of representative Anisoles.

\begin{tabular}{|c|c|c|c|c|c|c|c|}
\hline \multirow{3}{*}{ Reactant } & \multirow{3}{*}{ Product } & \multicolumn{4}{|c|}{ Thermal reaction } & \multicolumn{2}{|c|}{$\begin{array}{c}\text { Solvent free } \\
\text { reaction }\end{array}$} \\
\hline & & $\begin{array}{r}\text { RT } \\
\text { (h) }\end{array}$ & $\begin{array}{c}\text { Yield } \\
(\%) \\
\end{array}$ & $\begin{array}{r}\text { RT } \\
\text { (h) }\end{array}$ & $\begin{array}{c}\text { Yield } \\
(\%) \\
\end{array}$ & \multirow{2}{*}{$\begin{array}{c}\text { RT } \\
(\mathbf{m i n})\end{array}$} & $\begin{array}{c}\text { Yiel } \\
\text { d }\end{array}$ \\
\hline & & \multicolumn{2}{|c|}{ DCE } & \multicolumn{2}{|c|}{$\mathbf{A C N}$} & & $(\%)$ \\
\hline $1 \mathrm{a}$ & $2 \mathrm{a}$ & 6 & 40 & 6 & 57 & 30 & 36 \\
\hline $1 \mathrm{~b}$ & $2 b$ & 6 & 45 & 6 & 50 & 30 & 25 \\
\hline $1 \mathrm{c}$ & $2 c$ & 6 & 50 & 6 & 55 & 25 & 35 \\
\hline $1 d$ & $2 d$ & 6 & 42 & 6 & 40 & 30 & 31 \\
\hline $1 \mathrm{e}$ & $2 \mathrm{e}$ & 6 & 40 & 6 & 52 & 30 & 30 \\
\hline $1 \mathrm{f}$ & $2 \mathrm{f}$ & 6 & 44 & 6 & 42 & 25 & 37 \\
\hline $4 \mathrm{a}$ & $5 \mathrm{a}$ & 6 & 30 & 6 & 24 & 30 & 35 \\
\hline $4 \mathrm{~b}$ & $5 b$ & 6 & 60 & 6 & 58 & 25 & 38 \\
\hline $4 \mathrm{c}$ & $5 c$ & 6 & 57 & 6 & 62 & 30 & 33 \\
\hline $4 d$ & $5 d$ & 6 & 52 & 6 & 48 & 30 & 34 \\
\hline
\end{tabular}

${ }^{\mathrm{a}}$ Vilsmeier-Haack Formylation $=\left(\mathrm{DMF}+\mathrm{POCl}_{3}\right)(1: 1)$

Table 4. Spectroscopic data of Acetylation ${ }^{\mathrm{b}}$ Reactions of representative

\begin{tabular}{|c|c|c|c|c|c|c|c|}
\hline \multirow{3}{*}{ Reactant } & \multirow{3}{*}{ Product } & \multicolumn{4}{|c|}{ Thermal reaction } & \multicolumn{2}{|c|}{$\begin{array}{c}\text { Solvent } \\
\text { free } \\
\text { reaction }\end{array}$} \\
\hline & & $\begin{array}{l}\text { RT } \\
\text { (h) } \\
\end{array}$ & $\begin{array}{c}\text { Yield } \\
(\%)\end{array}$ & $\begin{array}{l}\text { RT } \\
\text { (h) } \\
\end{array}$ & $\begin{array}{c}\text { Yield } \\
(\%)\end{array}$ & \multirow{2}{*}{$\begin{array}{c}\text { RT } \\
(\mathbf{m i n})\end{array}$} & \multirow{2}{*}{$\begin{array}{c}\text { Yield } \\
(\%)\end{array}$} \\
\hline & & \multicolumn{2}{|c|}{ DCE } & \multicolumn{2}{|c|}{$\mathrm{ACN}$} & & \\
\hline $1 \mathrm{a}$ & $3 a$ & 6 & 50 & 6 & 42 & 30 & 37 \\
\hline $1 \mathrm{~b}$ & $3 \mathrm{~b}$ & 6 & 56 & 6 & 55 & 30 & 30 \\
\hline $1 \mathrm{c}$ & $3 \mathrm{c}$ & 6 & 40 & 6 & 55 & 25 & 30 \\
\hline $1 d$ & $3 d$ & 6 & 30 & 6 & 55 & 30 & 30 \\
\hline $1 \mathrm{e}$ & $3 \mathrm{e}$ & 6 & 40 & 6 & 40 & 30 & 30 \\
\hline 1f & $3 f$ & 6 & 43 & 6 & 53 & 25 & 40 \\
\hline $4 \mathrm{a}$ & $6 a$ & 6 & 63 & 6 & 52 & 30 & 37 \\
\hline $4 b$ & $6 \mathrm{~b}$ & 6 & 58 & 6 & 60 & 25 & 40 \\
\hline $4 \mathrm{c}$ & $6 c$ & 6 & 68 & 6 & 50 & 30 & 30 \\
\hline $4 d$ & $6 \mathrm{~d}$ & 6 & 56 & 6 & 46 & 30 & 35 \\
\hline
\end{tabular}
Anisoles.

\begin{tabular}{|c|c|c|c|}
\hline Compound & $\mathbf{M}^{+}$ & IR $\left(\mathrm{Cm}^{-1}\right)$ & NMR \\
\hline $3 a$ & 150 & $1690(\mathrm{C}=\mathrm{O})$ & $\begin{array}{l}\delta \quad 2.50\left(\mathrm{~s}, 3 \mathrm{H}, \mathrm{COCH}_{3}\right) \\
\left.3.85(\mathrm{~s}, 3 \mathrm{H}, \mathrm{OCH})_{3}\right) \\
7.08(\mathrm{~d}, 1 \mathrm{H}, \mathrm{Ar}) \\
7.90(\mathrm{~d}, 1 \mathrm{H}, \mathrm{Ar})\end{array}$ \\
\hline $3 b$ & 164 & $1690(\mathrm{C}=\mathrm{O})$ & $\begin{array}{ll}\delta & 2.16\left(\mathrm{~s}, 3 \mathrm{H}, \mathrm{CH}_{3}\right) \\
3.73 & \left.(\mathrm{~s}, 3 \mathrm{H}, \mathrm{OCH})_{3}\right) \\
6.76 & (\mathrm{~d}, 1 \mathrm{H}, \mathrm{Ar}) \\
7.71 & (\mathrm{~s}, 1 \mathrm{H}, \mathrm{Ar}) \\
7.55 & (\mathrm{~d}, 1 \mathrm{H}, \mathrm{Ar}) \\
\end{array}$ \\
\hline $3 c$ & 164 & $1690(\mathrm{C}=\mathrm{O})$ & $\begin{array}{ll}\delta & 2.35\left(\mathrm{~s}, 3 \mathrm{H}, \mathrm{CH}_{3}\right) \\
2.55\left(\mathrm{~s}, 3 \mathrm{H}, \mathrm{COCH}_{3}\right) \\
3.73\left(\mathrm{~s}, 3 \mathrm{H}, \mathrm{OCH}_{3}\right) \\
6.84(\mathrm{~d}, 1 \mathrm{H}, \mathrm{Ar}) \\
7.06(\mathrm{~d}, 1 \mathrm{H}, \mathrm{Ar}) \\
7.25(\mathrm{~s}, 1 \mathrm{H}, \mathrm{Ar}) \\
\end{array}$ \\
\hline 3d & 195 & $1690(\mathrm{C}=\mathrm{O})$ & $\begin{array}{l}\delta 2.55\left(\mathrm{~s}, 3 \mathrm{H}, \mathrm{COCH}_{3}\right) \\
\left.3.83(\mathrm{~s}, 3 \mathrm{H}, \mathrm{OCH})_{3}\right) \\
7.14(\mathrm{~d}, 1 \mathrm{H}, \mathrm{Ar}) \\
8.39(\mathrm{~d}, 1 \mathrm{H}, \mathrm{Ar}) \\
8.68(\mathrm{~s}, 1 \mathrm{H}, \mathrm{Ar}) \\
\end{array}$ \\
\hline $3 e$ & 184 & $1690(\mathrm{C}=\mathrm{O})$ & $\begin{array}{ll}\delta & 2.55\left(\mathrm{~s}, 3 \mathrm{H}, \mathrm{COCH}_{3}\right) \\
3.83(\mathrm{~s}, 3 \mathrm{H}, \mathrm{OCH} & \\
7.18 & (\mathrm{~d}, 1 \mathrm{H}, \mathrm{Ar}) \\
7.55 & (\mathrm{~d}, 1 \mathrm{H}, \mathrm{Ar}) \\
7.76 & (\mathrm{~s}, 1 \mathrm{H}, \mathrm{Ar}) \\
\end{array}$ \\
\hline $3 f$ & 200 & $1690(\mathrm{C}=\mathrm{O})$ & $\begin{array}{l}\delta \quad 2.55\left(\mathrm{~s}, 3 \mathrm{H}, \mathrm{COCH}_{3}\right) \\
\left.3.73(\mathrm{~s}, 3 \mathrm{H}, \mathrm{OCH})_{3}\right) \\
7.66(\mathrm{t}, 1 \mathrm{H}, \mathrm{Ar}) \\
7.77(\mathrm{t}, 1 \mathrm{H}, \mathrm{Ar}) \\
7.83(\mathrm{~d}, 1 \mathrm{H}, \mathrm{Ar}) \\
8.29(\mathrm{~d}, 1 \mathrm{H}, \mathrm{Ar}) \\
8.49(\mathrm{~d}, 1 \mathrm{H}, \mathrm{Ar}\end{array}$ \\
\hline
\end{tabular}

Table 1. Vilsmeier Haack Formylation Reactions with Anisoles and Pyridines.

Table 2. Vilsmeier Haack Acetylation Reactions with Anisoles and Pyridines.

${ }^{\mathrm{b}}$ Vilsmeier-Haack Acetylation $=\left(\mathrm{DMA}+\mathrm{POCl}_{3}\right)(1: 1)$

On comparison of VHR under classical and nonclassical conditions, it was observed that the reaction was completed in 6 hours under thermal conditions using dichloroethane (DCE) and acetonitrile (ACN) solvent, and within 25-30 minutes under solvent free conditions. This 


\section{Advanced Materials Letters www. vbripress.com/aml}

huge difference in the reaction time makes the author believe that non-classical method not only saves time and energy, but reduces the expenses and pollution.

Table 5. Spectroscopic data of Formylation ${ }^{\text {a }}$ Reactions of representative Pyridines.

\begin{tabular}{|c|c|c|c|}
\hline Compound & $\mathbf{M}^{+}$ & IR $\left(\mathrm{Cm}^{-1}\right)$ & NMR \\
\hline $5 a$ & 107 & $1690(\mathrm{C}=\mathrm{O})$ & $\begin{array}{l}\delta 7.50(\mathrm{t}, 1 \mathrm{H}, \mathrm{Ar}) \\
8.17(\mathrm{~d}, 1 \mathrm{H}, \mathrm{Ar}) \\
8.71(\mathrm{~d}, 1 \mathrm{H}, \mathrm{Ar}) \\
9.04(\mathrm{~s}, 1 \mathrm{H}, \mathrm{Ar}) \\
9.61(\mathrm{~s}, 1 \mathrm{H}, \mathrm{CHO})\end{array}$ \\
\hline $5 \mathbf{b}$ & 136 & $1690(\mathrm{C}=\mathrm{O})$ & $\begin{array}{l}\delta 2.37\left(\mathrm{~s}, 3 \mathrm{H}, \mathrm{CH}_{3}\right) \\
6.51\left(\mathrm{~s}, 2 \mathrm{H}, \mathrm{NH}_{2}\right) \\
6.72(\mathrm{~s}, 1 \mathrm{H}, \mathrm{Ar}) \\
8.57(\mathrm{~s}, 1 \mathrm{H}, \mathrm{Ar}) \\
9.61(\mathrm{~s}, 1 \mathrm{H}, \mathrm{CHO})\end{array}$ \\
\hline $5 c$ & 122 & $1690(\mathrm{C}=\mathrm{O})$ & $\begin{array}{l}55.85\left(\mathrm{~s}, 2 \mathrm{H}, \mathrm{NH}_{2}\right) \\
7.68(\mathrm{~s}, 1 \mathrm{H}, \mathrm{Ar}) \\
8.68(\mathrm{~s}, 1 \mathrm{H}, \mathrm{Ar}) \\
8.73(\mathrm{~s}, 1 \mathrm{H}, \mathrm{Ar}) \\
9.61(\mathrm{~s}, 1 \mathrm{H}, \mathrm{CHO})\end{array}$ \\
\hline 5d & 136 & $1690(\mathrm{C}=\mathrm{O})$ & $\begin{array}{l}\delta 2.32\left(\mathrm{~s}, 3 \mathrm{H}, \mathrm{CH}_{3}\right) \\
6.51(\mathrm{~s}, 2 \mathrm{H}, \mathrm{NH} 2) \\
7.80(\mathrm{~s}, 1 \mathrm{H}, \mathrm{Ar}) \\
8.54(\mathrm{~s}, 1 \mathrm{H}, \mathrm{Ar}) \\
9.61(\mathrm{~s}, 1 \mathrm{H}, \mathrm{CHO})\end{array}$ \\
\hline
\end{tabular}

${ }^{\mathrm{a}}$ Vilsmeier-Haack Formylation $=\left(\mathrm{DMF}+\mathrm{POCl}_{3}\right)(1: 1)$

Progress of the reaction is due to the heat energy generated from the mechanical energy when the reaction mixture is grinded in the mortar. Even though the yields are low, the reaction is completed in 25-30 minutes under solvent free conditions, suggesting that the present method could be employed for small scale Vilsmeier-Haack synthesis of formylation / acetylation reaction. Since the method is solvent free and saves time it could be considered as a green synthesis of Vilsmeier-Haack reactions.

Table 6. Spectroscopic data of Acetylation ${ }^{\text {b }}$ Reactions of representative Pyridines.

\begin{tabular}{|c|c|c|c|}
\hline Compound & $\mathbf{M}^{+}$ & IR $\left(\mathbf{C m}^{-1}\right)$ & $\begin{array}{c}\text { NMR } \\
\end{array}$ \\
\hline $6 a$ & 121 & $1690(\mathrm{C}=\mathrm{O})$ & $\begin{array}{c}\delta 2.55(\mathrm{~s}, 3 \mathrm{H}, \mathrm{COCH} 3) \\
7.68(\mathrm{t}, 1 \mathrm{H}, \mathrm{Ar}) \\
8.43(\mathrm{~d}, 1 \mathrm{H}, \mathrm{Ar}) \\
8.88(\mathrm{~d}, 1 \mathrm{H}, \mathrm{Ar}) \\
9.31(\mathrm{~s}, 1 \mathrm{H}, \mathrm{Ar})\end{array}$ \\
\hline $6 \mathbf{b}$ & 150 & $1690(\mathrm{C}=\mathrm{O})$ & $\begin{array}{c}\delta 2.55(\mathrm{~s}, 3 \mathrm{H}, \mathrm{COCH} 3) \\
2.37(\mathrm{~s}, 3 \mathrm{H}, \mathrm{NH} 2) \\
6.51(\mathrm{~s}, 2 \mathrm{H}, \mathrm{Ar}) \\
6.90(\mathrm{~s}, 1 \mathrm{H}, \mathrm{Ar}) \\
8.84(\mathrm{~s}, 1 \mathrm{H}, \mathrm{Ar})\end{array}$ \\
\hline 6c & 136 & $1690(\mathrm{C}=\mathrm{O})$ & $\begin{array}{cl}\delta & 2.55(\mathrm{~s}, 3 \mathrm{H}, \mathrm{COCH} 3) \\
& 5.85(\mathrm{~s}, 2 \mathrm{H}, \mathrm{NH} 2) \\
& 7.94(\mathrm{~s}, 1 \mathrm{H}, \mathrm{Ar}) \\
& 8.90(\mathrm{~d}, 1 \mathrm{H}, \mathrm{Ar})\end{array}$ \\
\hline 6d & 150 & $1690(\mathrm{C}=\mathrm{O})$ & $\begin{array}{ll}\delta & 2.32(\mathrm{~s}, 1 \mathrm{H}, \mathrm{Ar}) \\
& 2.55(\mathrm{~s}, 3 \mathrm{H}, \mathrm{COCH} 3) \\
& 6.51(\mathrm{~s}, 2 \mathrm{H}, \mathrm{NH} 2) \\
& 8.06(\mathrm{~s}, 1 \mathrm{H}, \mathrm{Ar}) \\
& 8.81(\mathrm{~s}, 1 \mathrm{H}, \mathrm{Ar})\end{array}$ \\
\hline
\end{tabular}

${ }^{\mathrm{b}}$ Vilsmeier-Haack Acetylation $=\left(\mathrm{DMA}+\mathrm{POCl}_{3}\right)(1: 1)$

\section{Conclusion}

In conclusion, we have successfully demonstrated Vilsmeier-Haack reaction with certain anisoles and pyridines under solvent free conditions. The present finding is more advantageous over solution phase reaction. It is conducted with economically cheap and readily available reagents. The reaction occurs under mild and environmentally safe conditions with a simple work up at room temperature. Thus, it is believed that the present work is a major break through in the area of VilsmeierHaack synthesis.

\section{Acknowledgements}

Professor K.C. Rajanna is kindly acknowledged for his constant encouragement and discussion throughout the project. The author thanks Nawab Shah Alam Khan Center for Post Graduate Studies and Research, Hyderabad, for providing necessary research facilities to the Chemistry Department.

\section{Keywords}

Vilsmeier-Haack reaction, anisoles, pyridines, dimethylformamide, dimethylacetamide.

Received: 02 December 2019

Revised: 19 December 2019

Accepted: 23 December 2019

\section{References}

1. Oleg, K.F.; Victor, I.; Markov; Svetlana, A.; Varenichenko, Victor, V.; Dotsenko; Alexander, V.; Mazepa; Tetrahedron, 2015, 71, 5554.

2. Jairo, Q.; Jorge, T.; Braulio, I.; Rodrigo, A.; Manuel, N.; Justo, C.; Tetrahedron Letters, 2008, 49, 2689.

3. Rajanna, K.C.; Florence, S.; Mir Moazzam, A.; Saiprakash, P.K.; Tetrahedron, 1996, 52, 10, 3669.

4. Miguel, A.A.; J. Ignacio, F.; Carmen, A.; J Carlos, M.; Mercedes, V.; Tetrahedron, 1993, 49, 10997.

5. Yi Luo; Ping, Z.; Xiao, H.Z.; Qiu, L.L.; Ye Na, C.; Chinese Chemical Letters, 2008, 19, 383.

6. Jairo, Q.; Yurina, D.; Braulio, I.; Rodrigo, A.; Manuel, N.; Justo, C.; Tetrahedron Letters, 2010, 51, 2928.

7. Ge, M.; Yao Wu, S.; Rui, Z.; Nan, B.; Chinese Chemical Letters, 2011, 22, 1043.

8. Radhey, M.S.; Neha, S.; Ritush, K.; Mrityunjaya, A.; Shraddha, U.; Tetrahedron, 2012, 68, 10318

9. John, T.G. Edith, J.B.; Melissa, D.S.; Matthew, B.C.; Jonathan, E.H.; Anastasia, K.; Daniel, C.F.; Ben, C.G.; Kristin, L.S.; Matt, J.K.; Timothy, M.S.; Rene, P.F.K.; Raymond, N.D.; James, A.S.; Tetrahedron, 2008, 64, 5246.

10. Nidhin, P.; Shanmugam, M.; Domino; Tetrahedron Letters, 2011, 52,3743 .

11. Thomas, A.D.; Asokan, C.V.; Tetrahedron Letters, 2002, 43, 2273.

12. Thomas, A.D.; Josemin; Asokan, C.V.; Tetrahedron, 2004, 60, 5069

13. Christina, B.; Galina, R.; Tatyana, R.; Yuri, G.; Vladimir, R.; Tetrahedron, 2008, 64, 9191.

14. Shi-Han, L.; Wan-Ping, Y.; Henry, J.T.; Chien-Shu, C.; Fung, F.W.; Tetrahedron, 2015, 71, 6749.

15. Damodiran, M.; Panneer Selvam, N.; Paramasivan, T.; Perumal; Tetrahedron Letters, 2009, 50, 5474.

16. Daulat, B.K.; Su Hui, Y.; Suk Hee, C.; Chao Z.; Won-Jea, C.; Tetrahedron, 2012, 68, 250.

17. Sousuke, U.; Katsuhiko, M.; Hideo, T.; Tetrahedron, 2012, 68 4588.

18. Hojat, V.; Meral, A.; Pegah, D.T.; Effat, A.; Ali Reza, F.; Chimie., 2014, 17, 305

19. Fung, F.W.; Yu-Ying, H.; Tetrahedron, 2011, 67, 3863.

20. Yi Yi, W.; Lei Ming, Y.; Qi Xu, C.; Wei, K.S.; Chinese Chemical Letters, 2012, 23, 911.

21. Yi L.; Ping, Z.; Xiao, H.Z.; Qiu, L.L.; Ye Na, C.; Chinese Chemical Letters, 2008, 19, 383

22. (a) Mir, M.A.; Tasneem, M.; Rajanna, K.C.; Saiprakash, P.K.; Synlett, 2001, 2, 251; (b) Rajanna, K.C.; Mir, M.A.; Sana, S.; Tasneem, M.; Saiprakash, P.K.; Journal of Dispersion Science and Technology, 2004, 25(1), 17. 